\title{
Certain relations between Bessel and Whittaker functions related to some diagonal and block-diagonal $3 \times 3$-matrices
}

\author{
I. A. Shilin ${ }^{\mathrm{a}, \mathrm{b}}$, Junesang $\mathrm{Choi}^{\mathrm{c}, *}$ \\ ${ }^{a}$ Department of Mathematics, Sholokhov Moscow State University for the Humanities, Verhnya Radishevskaya 16-18, Moscow 109240, \\ Russia. \\ ${ }^{b}$ Department of Energetics, University of Economics and Energetics, Kirovogradskaya ul. 11-1, Moscow 117587. \\ ${ }^{c}$ Department of Mathematics, Dongguk University, Gyeongju 38066, Republic of Korea.
}

Communicated by Y. J. Cho

\begin{abstract}
The authors derive the matrix elements of the linear operators which appear under the representation of the group $\mathrm{SO}(2,1)$ and correspond to some diagonal or block-diagonal matrices belonging to the above group. Then, by applying these matrix elements, that is, from a group theoretical point of view, the authors show how certain interesting integral and series representations of the Whittaker function of the second kind and some formulas for the (basic and modified) Bessel functions can be obtained. A special case of one of the results presented here is indicated to be also a special one of a known formula. (C)2017 All rights reserved.
\end{abstract}

Keywords: Whittaker function, Bessel functions, Macdonald function, semisimple Lie group SO $(2,1)$, matrix elements of representation.

2010 MSC: 33C10, 33C80, 33B15, 33C05.

\section{Introduction and preliminaries}

Throughout this paper, we denote the sets of integers, real numbers, and complex numbers by $\mathbb{Z}, \mathbb{R}$, and $\mathbb{C}$, respectively. It is well-known that the irreducible representations of the three-dimensional Lorentz group $\mathrm{SO}(2,1)$ can be constructed in the space of $\sigma$-homogeneous infinitely differentiable functions $(\sigma \in$ $\mathbb{C} \backslash \mathbb{Z}$ ) defined on the cone

$$
\Lambda:=\left\{\left(x_{1}, x_{2}, x_{3}\right) \in \mathbb{R}^{3} \mid x_{1}^{2}-x_{2}^{2}-x_{3}^{2}=0\right\} .
$$

For the fixed $\sigma$ with $-1<\mathfrak{R}(\sigma)<0$, we denote this space by $\mathfrak{D}$ and the analogous space consisting of $\sigma$-homogeneous functions when $\sigma:=-\sigma-1$ by $\mathfrak{D}^{\bullet}$. The linear operator of the space $\mathfrak{D}$, arising in this case and corresponding to the Lorentz group element $g$, can be written in the form $T(g)[f(x)]=f\left(g^{-1} x\right)$. Since the functions belonging to $\mathfrak{D}$ are homogeneous, we can realize the representation $T_{\sigma}$ in the space of the

\footnotetext{
*Corresponding author

Email addresses: ilyashilin@li.ru (I. A. Shilin), junesang@mail.dongguk.ac.kr (Junesang Choi)
} 
restrictions $\hat{f}$ of functions $f \in \mathfrak{D}$ to any conic section $\gamma$, because $\hat{f}(\xi)=t^{-\sigma} f(t \xi)=t(x)$ for $\xi \in \gamma$. Vilenkin and Shleinikova [8] considered the following three sections of $\Lambda$ : The circle $\gamma_{1}: x_{1}=1$; the hyperbola $\gamma_{2}: x_{2}= \pm 1$; and the parabola $\gamma_{3}: x_{1}+x_{2}=1$. They introduced three bases in $\mathfrak{D}$, which consist of the eigenfunctions of the restrictions of the representation $T$ to the subgroups acting transitively on the above conic sections, respectively, and obtained formulas for 'matrix elements' of the linear operator, which transforms the 'circle' basis into the 'parabolic' basis, and some 'matrix elements' of the linear operator, which transforms the 'parabolic' basis into the 'hyperbolic' basis. We denote these bases by $B_{1}, B_{2}$, and $\mathrm{B}_{3}$, respectively. Namely,

$$
\begin{aligned}
& \mathrm{B}_{1}:=\left\{\mathrm{f}_{\mathrm{k}}(x):=x_{1}^{\sigma-k}\left(x_{1}+\mathbf{i} x_{2}\right)^{\mathrm{k}} \mid \mathrm{k} \in \mathbb{Z}\right\}, \\
& \mathrm{B}_{2}:=\left\{\mathrm{f}_{\rho, \pm}^{*}(\mathrm{x}):=\left(\mathrm{x}_{1}\right)_{ \pm}^{\sigma-\mathbf{i} \rho}\left(\mathrm{x}_{1}+\mathrm{x}_{2}\right)^{\mathbf{i} \rho} \mid \rho \in \mathbb{R}\right\}, \\
& \mathrm{B}_{3}:=\left\{\mathrm{f}_{\lambda}^{* *}(x):=\left(x_{1}+x_{2}\right)^{\sigma} \exp \left(\frac{\mathbf{i} \lambda x_{3}}{x_{1}+x_{2}}\right) \mid \lambda \in \mathbb{R}\right\},
\end{aligned}
$$

where $\left(x_{1}\right)_{ \pm}^{\lambda}$ is equal to $\left( \pm x_{1}\right)^{\lambda}$ in case of $\operatorname{sign} x_{1}= \pm 1$ and equal to zero otherwise.

Shilin [3] determined the bilinear functionals $D_{i}: \mathfrak{D}^{2} \longrightarrow \mathbb{C}$ by formula

$$
(u, v) \longmapsto \iint_{\gamma_{i}^{2}} k(x, \hat{x}) u(x) v(\hat{x}) \mathrm{d} x \mathrm{~d} \hat{x}, \quad(i=1,2,3),
$$

where $(\mathrm{dx})_{\gamma_{i}}$ is the $\mathrm{H}_{i}$-invariant measure and $H_{i}$ denotes the subgroup acting transitively on $\gamma_{i}$. Shilin [3] found (up to the constant multiplier $C_{i}$, respectively) the restrictions of the kernel $k(x, \hat{x})$ to the above sections such that $D_{1}=D_{2}=D_{3}$. By using the equality $D_{i}(u, v)=D_{j}(u, v)(i \neq j)$ for $u \in B_{p}$ and $v \in B_{q}$ $(p \neq q)$, Shilin [3] obtained some formulas for the Whittaker functions, which were able to compute the fractions $\frac{C_{i}}{C_{j}}$ from some simple initial conditions.

Shilin and Choi [4-6] have considered two spaces $\mathfrak{D}$ and $\mathfrak{D}^{\prime}$ for groups $\mathrm{SO}(3,1)$ and $\mathrm{SO}(2,2)$ and determined the bilinear functional $F_{i}: \mathfrak{D} \times \mathfrak{D}^{\prime}$ by the rule

$$
(\mathrm{u}, v) \longmapsto \int_{\gamma_{i}} \mathrm{u}(\mathrm{x}) v(\mathrm{x})(\mathrm{dx})_{\gamma_{i}},
$$

where $\gamma_{i}$ denote the spherical, parabolic and hyperbolic sections of the corresponding cones. Shilin and Choi [4-6] have computed the matrix elements of the basis transform operators which together with the generalized Mehler-Fock transform, have been used to obtain some formulas for Legendre functions in case of $\mathrm{SO}(3,1)$ and Whittaker functions in case of $\mathrm{SO}(2,2)$.

In this paper we return to consider the group $\mathrm{SO}(2,1)$ and compute the matrix elements of the operators $\mathrm{T}^{\bullet}(\mathrm{g})$ for some fixed group elements $\mathrm{g}$. We apply these matrix elements to present some integral and series representations of the Whittaker function of the second kind and certain formulas for the Bessel functions and single- and double-variable formulas for the Macdonald functions.

Lemma 1.1. In case of $\mathfrak{D}^{\prime}=\mathfrak{D}^{\bullet}$, the functionals $\mathrm{F}_{1}, \mathrm{~F}_{2}$, and $\mathrm{F}_{3}$ coincide. For any $\mathrm{g} \in \mathrm{SO}(2,1)$, the functional $\mathrm{F}_{\mathrm{i}}$ is invariant with respect to the pair $(\mathrm{T}(\mathrm{g}), \mathrm{T} \bullet(\mathrm{g}))$ of operators arising in the corresponding spaces, that is, $\mathrm{F}_{\mathrm{i}}\left(\mathrm{T}(\mathrm{g})[\mathrm{u}], \mathrm{T}^{\bullet}(\mathrm{g})[v]\right)=\mathrm{F}_{\mathrm{i}}(\mathrm{u}, \mathrm{v})$.

Proof. We can describe the above conic sections by using a single parameter. In particular,

$$
\gamma_{1}=\{(1, \cos \varphi, \sin \varphi) \mid \varphi \in[-\pi, \pi)\},
$$

and

$$
\gamma_{2}=\{(\cosh s, \pm 1, \sinh s) \mid s \in \mathbb{R}\}
$$


It is obvious that $(\mathrm{dx})_{\gamma_{1}}=\mathrm{d} \varphi$ and $(\mathrm{d} x)_{\gamma_{2}}=\mathrm{d}$. Then we have

$$
\begin{aligned}
\mathrm{F}_{1}(u, v) & =\int_{-\pi}^{\pi} u(\varphi) v(\varphi) d \varphi \\
& =\int_{-\pi}^{\pi} u\left(|\sec \varphi|, \pm 1, \frac{\sin \varphi}{|\cos \varphi|}\right) v\left(|\sec \varphi|, \pm 1, \frac{\sin \varphi}{|\cos \varphi|}\right) \frac{\mathrm{d} \varphi}{|\sec \varphi|}
\end{aligned}
$$

Setting $\varphi=\arccos \frac{1}{|\cosh |}$, we acquire $\mathrm{d} \varphi=\frac{\mathrm{ds}}{|\cosh s|}$. We therefore have $\mathrm{F}_{1}(u, v)=\mathrm{F}_{2}(u, v)$. A similar argument can show that $\mathrm{F}_{1}(\mathrm{u}, \mathrm{v})=\mathrm{F}_{3}(\mathrm{u}, \mathrm{v})$.

The group $\mathrm{SO}(2,1)$, as a semisimple Lie group, admits the Cartan decomposition $\mathrm{SO}(2,1)=\mathrm{KAK}$, where $\mathrm{K}$ is a maximal compact subgroup and $A$ is a maximal Abelian group. These groups consist of matrices

$$
g_{1}(\psi):=\left(\begin{array}{rrr}
1 & 0 & 0 \\
0 & \cos \psi & -\sin \psi \\
0 & \sin \psi & \cos \psi
\end{array}\right), \quad \text { and } \quad g_{2}(t):=\left(\begin{array}{rrr}
\cosh t & \sinh t & 0 \\
\sinh t & \cosh t & 0 \\
0 & 0 & 1
\end{array}\right)
$$

respectively. Then $g_{1}^{-1}(\psi)=g_{1}(-\psi), g_{2}^{-1}(t)=g_{2}(-t)$ and

$$
\begin{aligned}
& \mathrm{F}_{i}\left(\mathrm{~T}\left(\mathrm{~g}_{1}(\psi)\right)[u], \mathrm{T}^{\bullet}\left(\mathrm{g}_{1}(\psi)\right)[v]\right)=\mathrm{F}_{1}\left(\mathrm{~T}\left(\mathrm{~g}_{1}(\psi)\right)[u], \mathrm{T}^{\bullet}\left(\mathrm{g}_{1}(\psi)\right)[v]\right) \\
& =\int_{-\pi}^{\pi} u(\varphi-\psi) u(\varphi-\psi) d \varphi=\int_{-\pi-\psi}^{\pi-\psi} u(z) u(z) d(z+\psi)=F_{1}(u, v), \\
& \mathrm{F}_{\mathfrak{i}}\left(\mathrm{T}\left(\mathrm{g}_{2}(\mathrm{t})\right)[\mathrm{u}], \mathrm{T}^{\bullet}\left(\mathrm{g}_{2}(\mathrm{t})\right)[v]\right)=\mathrm{F}_{1}\left(\mathrm{~T}\left(\mathrm{~g}_{2}(\mathrm{t})\right)[\mathrm{u}], \mathrm{T}^{\bullet}\left(\mathrm{g}_{2}(\mathrm{t})\right)[v]\right) \\
& =\int_{-\pi}^{\pi} u\left(1, \frac{\cosh t \cos \varphi-\sinh t}{\cosh t-\sinh t \cos \varphi}, \frac{\sin \varphi}{\cosh t-\sinh t \cos \varphi}\right) \\
& \times v\left(1, \frac{\cosh t \cos \varphi-\sinh t}{\cosh t-\sinh t \cos \varphi}, \frac{\sin \varphi}{\cosh t-\sinh t \cos \varphi}\right) \frac{d \varphi}{\cosh t-\sinh t \cos \varphi} .
\end{aligned}
$$

Choosing here the new variable $\theta:=\arctan \frac{\sin \varphi}{\cosh t \cos \varphi-\sin t}$, we obtain

$$
\mathrm{F}_{1}\left(\mathrm{~T}\left(\mathrm{~g}_{2}(\mathrm{t})\right)[\mathrm{u}], \mathrm{T}^{\bullet}\left(\mathrm{g}_{2}(\mathrm{t})\right)[v]\right)=\mathrm{F}_{1}(\mathrm{u}, \mathrm{v}) .
$$

\section{Formulas related to the matrix $\operatorname{diag}(1,-1,-1)$}

Let us consider the matrix elements $t_{\lambda \hat{\lambda}}(g)$ of the representation $T^{\bullet}$ with respect to the basis $B_{2}$. Since

$$
T^{\bullet}(g)\left[f_{\lambda}^{* *}(x)\right]=\int_{-\infty}^{+\infty} t_{\lambda \hat{\lambda}}(g) f_{\hat{\lambda}}^{* *}(x) d \hat{\lambda}
$$

we have

$$
F_{i}\left(T^{\bullet}(g)\left[f_{\lambda}^{* *}\right], f_{\tau}^{* *}\right)=\int_{-\infty}^{+\infty} t_{\lambda \mu}(g) F_{j}\left(f_{\hat{\lambda}}^{* *}, f_{\tau}^{* *}\right) d \hat{\lambda}
$$

for any $i$ and $j$. Taking

$$
F_{3}\left(f_{\hat{\lambda}}^{* *}, f_{\tau}^{* *}\right)=\int_{-\infty}^{+\infty} \exp (\mathbf{i}[\mu+\tau] y) d y=\delta(\mu+\tau)
$$


where $\delta(\mu+\tau)$ is the $(-\tau)$-delayed Dirac delta function, we obtain

$$
F_{i}\left(T^{\bullet}(g)\left[f_{\lambda}^{* *}\right], f_{\tau}^{* *}\right)=\int_{-\infty}^{+\infty} t_{\lambda \mu}(g) \delta(\mu+\tau) d \hat{\lambda}=2 \pi t_{\hat{\lambda},-\tau}(g) .
$$

Thus we have

$$
t_{\lambda \hat{\lambda}}(g)=\frac{1}{2 \pi} F_{i}\left(T^{\bullet}(g)\left[f_{\lambda}^{* *}\right], f_{-\hat{\lambda}}^{* *}\right) .
$$

Let us note that the matrix $\left(a_{i j}\right)$ belongs to $\mathrm{SO}(s, t)$, if and only if

$$
\sum_{j=1}^{t} a_{i j} a_{\hat{i} j}-\sum_{j=t+1}^{s+t} a_{i j} a_{\hat{i} j}= \begin{cases}0, & \text { if } i \neq \hat{i}, \\ 1, & \text { if } i=\hat{i} \leqslant t \\ -1, & \text { if } i=\hat{i}>t .\end{cases}
$$

In particular, $\operatorname{diag}(1,-1,-1) \in \mathrm{SO}(2,1)$. In the next lemma we obtain the matrix elements

$$
t_{\lambda \hat{\lambda}}(\operatorname{diag}(1,-1,-1))
$$

of which the case $\lambda \hat{\lambda} \neq 0$ can be expressed in terms of (basic and modified) Bessel functions.

Lemma 2.1. The matrix elements $t_{\lambda \hat{\lambda}}(\operatorname{diag}(1,-1,-1))$ are given as follows:

$\lambda \hat{\lambda} \neq 0$.

$$
t_{\lambda \hat{\lambda}}(\operatorname{diag}(1,-1,-1))= \begin{cases}\frac{\cos (\pi \sigma)}{2}\left(\frac{\hat{\lambda}}{\lambda}\right)^{\sigma+\frac{1}{2}}\left[J_{-2 \sigma-1}(2 \sqrt{\lambda \hat{\lambda}})-J_{2 \sigma+1}(2 \sqrt{\lambda \hat{\lambda}})\right], & \text { if } \lambda \hat{\lambda}>0, \\ -\frac{\sin (\pi \sigma)}{\pi}\left|\frac{\hat{\lambda}}{\lambda}\right|^{\sigma+\frac{1}{2}} K_{2 \sigma+1}(2 \sqrt{-\lambda \hat{\lambda}}), & \text { if } \lambda \hat{\lambda}<0 .\end{cases}
$$

$\boldsymbol{\square} \lambda \hat{\lambda}=0, \frac{1}{2} \delta_{\hat{\lambda}, 0}-1<\mathfrak{R}(\sigma)<-\frac{1}{2} \delta_{\lambda, 0}$.

$$
\mathrm{t}_{\lambda \hat{\lambda}}(\operatorname{diag}(1,-1,-1))= \begin{cases}-|\lambda|^{2 \sigma-1} \pi^{-1} \sin (\pi \sigma) \Gamma(2 \sigma+1), & \text { if } \lambda \neq 0 \\ -|\hat{\lambda}|^{2 \sigma+1} \pi^{-1} \sin (\pi \sigma) \Gamma(-2 \sigma-1), & \text { if } \hat{\lambda} \neq 0 .\end{cases}
$$

Here $\delta_{p, q}$ is the Kronecker symbol.

Proof. Indeed, we have $\gamma_{3}=\left\{\left(\frac{1+y^{2}}{2}, \frac{1-y^{2}}{2}, y\right) \mid y \in \mathbb{R}\right\}$ and $(d x)_{\gamma_{3}}=d y$ (see $[3,8]$ ).

Since $T^{\bullet}$ is a group homomorphism, in view of Lemma 1.1, we have

$$
\begin{aligned}
t_{\lambda \hat{\lambda}}(g) & =\frac{1}{2 \pi} F_{i}\left(T_{\sigma}(g)\left[f_{\lambda}^{* *}\right], f_{-\hat{\lambda}}^{* *}\right) \\
& =\frac{1}{2 \pi} F_{i}\left(T_{\sigma}\left(g^{-1}\right) T_{\sigma}(g)\left[f_{\lambda}^{* *}\right], T_{\sigma}\left(g^{-1}\right)\left[f_{-\hat{\lambda}}^{* *}\right]\right) \\
& =\frac{1}{2 \pi} F_{i}\left(f_{\lambda}^{* *}, T_{\sigma}\left(g^{-1}\right)\left[f_{-\hat{\lambda}}^{* *}\right]\right) .
\end{aligned}
$$

Since

$$
[\operatorname{diag}(1,-1,-1)]^{-1}=\operatorname{diag}(1,-1,-1),
$$

and

we have

$$
\left.f_{-\hat{\lambda}}^{* *}\right|_{\gamma_{3}}(\operatorname{diag}(1,-1,-1) x)=y^{2 \sigma} \exp \left(\frac{i \hat{\lambda}}{y}\right)
$$

$$
\begin{aligned}
t_{\lambda \hat{\lambda}}(\operatorname{diag}(1,-1,-1)) & =\frac{1}{2 \pi} F_{3}\left(f_{\lambda}^{* *}, T_{\sigma}(\operatorname{diag}(1,-1,-1))\left[f_{-\hat{\lambda}}^{* *}\right]\right) \\
& =\frac{1}{\pi} \int_{0}^{+\infty} y^{2 \sigma} \cos \left(\lambda y+\frac{\hat{\lambda}}{y}\right) d y
\end{aligned}
$$


The following formulas may be used:

- $\lambda \hat{\lambda}>0$. (See, e.g., [2, Entry 2.5.24.4]).

$$
\begin{aligned}
& \int_{0}^{+\infty} x^{\alpha-1} \cos \left(a x+\frac{b}{x}\right) d x=\frac{\pi}{2}\left(\frac{b}{a}\right)^{\frac{\alpha}{2}} \times \sin \left(\frac{\alpha \pi}{2}\right)\left[J_{-\alpha}(2 \sqrt{a b})-J_{\alpha}(2 \sqrt{a b})\right], \\
& (a, b>0 ;|\Re(\alpha)|<1) .
\end{aligned}
$$

- $\lambda \hat{\lambda}<0$. (See, e.g., [2, Entry 2.5.24.7]).

$$
\int_{0}^{+\infty} x^{\alpha-1} \cos \left(a x-\frac{b}{x}\right) d x=2\left(\frac{b}{a}\right)^{\frac{\alpha}{2}} \cos \left(\frac{\alpha \pi}{2}\right) K_{\alpha}(2 \sqrt{a b}), \quad(a, b>0 ;|\Re(\alpha)|<1) .
$$

$\lambda \neq 0$ and $\hat{\lambda}=0$. (See, e.g., [2, Entry 2.5.3.10]).

$$
\int_{0}^{+\infty} x^{\alpha-1} \cos (b x) d x=b^{-\alpha} \cos \left(\frac{\alpha \pi}{2}\right) \Gamma(\alpha), \quad(b>0 ; 0<\mathfrak{R}(\alpha)<1) .
$$

$\lambda=0$ and $\hat{\lambda} \neq 0$.

Substitute $y=z^{-1}$ and use the formula (2.1). 2.2 .

We obtain two similar integral representations of the Whittaker function $W_{\mu, v}$ asserted by Theorem

Theorem 2.2. The following integral representations for $W_{\mu, \nu}$ hold true:

$\lambda>0$.

$$
\begin{gathered}
\frac{\cos (\pi \sigma)}{2} \int_{0}^{+\infty} \hat{\lambda}^{-\frac{1}{2}} W_{-k, \sigma+\frac{1}{2}}(2 \hat{\lambda})\left[J_{-2 \sigma-1}(2 \sqrt{\lambda \hat{\lambda}})-J_{2 \sigma+1}(2 \sqrt{\lambda \hat{\lambda}})\right] d \hat{\lambda} \\
-\frac{\pi \Gamma(-k-\sigma) \sin (\pi \sigma)}{\Gamma(k-\sigma)} \int_{0}^{+\infty} \hat{\lambda}^{-\frac{1}{2}} W_{k, \sigma+\frac{1}{2}}(2 \hat{\lambda}) K_{2 \sigma+1}(2 \sqrt{\lambda \hat{\lambda}}) d \hat{\lambda} \\
=(-1)^{k} \lambda^{-\frac{1}{2}} W_{-k, \sigma+\frac{1}{2}}(2 \lambda) .
\end{gathered}
$$

$\lambda<0$.

$$
\begin{aligned}
& \frac{\cos (\pi \sigma)}{2} \int_{0}^{+\infty} \hat{\lambda}^{-\frac{1}{2}} W_{k, \sigma+\frac{1}{2}}(2 \hat{\lambda})\left[J_{-2 \sigma-1}(2 \sqrt{-2 \lambda \hat{\lambda}})-J_{2 \sigma+1}(2 \sqrt{-2 \lambda \hat{\lambda}})\right] d \hat{\lambda} \\
& -\frac{\sin (\pi \sigma) \Gamma(k-\sigma)}{\pi \Gamma(-k-\sigma)} \int_{0}^{+\infty} \hat{\lambda}^{-\frac{1}{2}} K_{2 \sigma+1}(2 \sqrt{-\lambda \hat{\lambda}}) W_{-k, \sigma+\frac{1}{2}}(2 \hat{\lambda}) d \hat{\lambda} \\
& =(-1)^{k}|\lambda|^{-\frac{1}{2}} W_{k, \sigma+\frac{1}{2}}(2|\lambda|) .
\end{aligned}
$$


$\lambda=0,-1<\Re(\sigma)<-\frac{1}{2}$

$$
\begin{gathered}
\pi^{-1} \sin (\pi \sigma) \Gamma(-2 \sigma-1)\left(\{\Gamma(-\sigma-k)\}^{-1} \int_{0}^{+\infty} \hat{\lambda}^{\sigma} W_{-k, \sigma+\frac{1}{2}}(2 \hat{\lambda}) \mathrm{d} \hat{\lambda}\right. \\
\left.+\{\Gamma(-\sigma+k)\}^{-1} \int_{0}^{+\infty} \hat{\lambda}^{\sigma} \mathrm{W}_{\mathrm{k}, \sigma+\frac{1}{2}}(2 \hat{\lambda}) \mathrm{d} \hat{\lambda}\right) \\
=(-1)^{\mathrm{k}} 2^{2 \sigma+1}(-2 \sigma-1)^{-1}\{\mathrm{~B}(\mathrm{k}-\sigma,-\mathrm{k}-\sigma)\}^{-1} .
\end{gathered}
$$

Proof. By using the connection $f_{\lambda}^{* *}(x)=\sum_{k \in \mathbb{Z}} c_{\lambda k} f_{k}(x)$ between the bases $B_{1}$ and $B_{3}$, we have

$$
\begin{aligned}
T^{\bullet}(\operatorname{diag}(1,-1,-1))\left[f_{\lambda}^{* *}(x)\right] & =\int_{-\infty}^{+\infty} t_{\lambda \hat{\lambda}}(\operatorname{diag}(1,-1,-1)) f_{\hat{\lambda}}^{* *}(x) d \hat{\lambda} \\
& =\sum_{k \in \mathbb{Z}}\left(\int_{-\infty}^{+\infty} t_{\lambda \hat{\lambda}}(\operatorname{diag}(1,-1,-1)) c_{\hat{\lambda} k} d \hat{\lambda}\right) f_{k}(x) .
\end{aligned}
$$

It is noted here that the 'matrix elements' of the linear operator that transforms $B_{1}$ into $B_{2}$ were computed for $\lambda \neq 0$ in [8]:

$$
\mathbf{c}_{\lambda k}=|\lambda|^{-\sigma-1}\{\Gamma(-\sigma-k \operatorname{sign} \lambda)\}^{-1} W_{-k \operatorname{sign} \lambda, \sigma+\frac{1}{2}}(2|\lambda|) .
$$

It is easy to see that

$$
\begin{aligned}
c_{0 k}=\frac{1}{2^{\sigma+1} \pi} F_{3}\left(f_{0}^{* *}, f_{-k}\right) & =\frac{1}{2^{\sigma+1} \pi} \int_{-\infty}^{+\infty}(1-\mathbf{i} y)^{\sigma-k}(1+\mathbf{i} y)^{\sigma+k} d y \\
& =2^{\sigma+1}(-2 \sigma-1)^{-1}\{B(k-\sigma,-k-\sigma)\}^{-1}, \quad \text { (see, e.g., [2, Entry 2.2.6.31]). }
\end{aligned}
$$

Let $V_{ \pm 1}$ be the linear subspace in $\mathfrak{D}^{\bullet}$ and consist of the zero function and the eigenfunctions of the operator $T^{\bullet}(\operatorname{diag}(1,-1,-1))$ whose corresponding eigenvalues are \pm 1 . It is easy to see that $f_{k} \in V_{(-1)^{k}}$, the 'infinite matrix' $\left(t_{k \hat{k}}(\operatorname{diag}(1,-1,-1))\right.$ of the operator $T^{\bullet}(\operatorname{diag}(1,-1,-1))$ with respect to the basis $B_{1}$ is diagonal and moreover, $t_{k \hat{k}}(\operatorname{diag}(1,-1,-1))=(-1)^{k}$. Then we have

$$
\begin{aligned}
T^{\bullet}(\operatorname{diag}(1,-1,-1))\left[f_{\lambda}^{* *}\right] & =T^{\bullet}(\operatorname{diag}(1,-1,-1))\left[\sum_{k \in \mathbb{Z}} c_{\lambda k} f_{k}\right] \\
& =\sum_{k \in \mathbb{Z}} T^{\bullet}(\operatorname{diag}(1,-1,-1))\left[f_{k}\right]=\sum_{k \in \mathbb{Z}}(-1)^{k} c_{\lambda k} f_{k} .
\end{aligned}
$$

From (2.5) and (2.6) we have

$$
\int_{-\infty}^{0} t_{\lambda \hat{\lambda}}(\operatorname{diag}(1,-1,-1)) c_{\hat{\lambda} k} d \hat{\lambda}+\int_{0}^{+\infty} t_{\lambda \hat{\lambda}}(\operatorname{diag}(1,-1,-1)) c_{\hat{\lambda} k} d \hat{\lambda}=(-1)^{k} c_{\lambda k},
$$

which immediately yields (2.2).

A similar argument can establish (2.3) and (2.4) whose proof details are omitted. 
Theorem 2.2 allows to obtain the following representation of the Macdonald function of doublevariable as a linear combination of the Macdonald and modified Struve functions.

Corollary 2.3. The following double-variable formulas hold true: For $\lambda>0$,

$$
\begin{aligned}
\mathrm{K}_{\sigma+\frac{1}{2}}(2 \lambda)= & \left(\frac{\sin (2 \pi \sigma)}{4}+1\right) \mathrm{K}_{\sigma+\frac{1}{2}}(\lambda) \\
& +\left(\frac{\pi \cos (\pi \sigma)}{4}-\frac{\pi}{8 \cos (\pi \sigma)}\right)\left[\mathbf{L}_{\sigma+\frac{1}{2}}(\lambda)-\mathbf{L}_{-\sigma-\frac{1}{2}}(\lambda)\right],
\end{aligned}
$$

where

$$
K_{v}(x):=\sqrt{\frac{\pi}{2 x}} W_{0, v}(2 x), \quad \text { and } \quad W_{\mu, v}(x)=W_{\mu,-v}(x) .
$$

Proof. The special case of (2.2) when $k=0$ can be rewritten as follows:

$$
\begin{array}{r}
\frac{\cos (\pi \sigma)}{2} \int_{0}^{+\infty} K_{\sigma+\frac{1}{2}}(\hat{\lambda})\left[J_{-2 \sigma-1}(2 \sqrt{\lambda \hat{\lambda}})-J_{2 \sigma+1}(2 \sqrt{\lambda \hat{\lambda}})\right] d \hat{\lambda} \\
-\frac{\sin (\pi \sigma)}{\pi} \int_{0}^{+\infty} K_{\sigma+\frac{1}{2}}(\hat{\lambda}) K_{2 \sigma+1}(2 \sqrt{\lambda \hat{\lambda}}) d \hat{\lambda}=K_{\sigma+\frac{1}{2}}(\lambda) .
\end{array}
$$

Use the following known formulas (see, e.g., [1, Entry 6.516.3] and [1, Entry 6.516.9], respectively):

$$
\int_{0}^{+\infty} J_{2 v}(a \sqrt{x}) K_{v}(b x) d x=\frac{\pi}{2}\left[I_{v}\left(\frac{a^{2}}{4 b}\right)-L_{v}\left(\frac{a^{2}}{4 b}\right)\right], \quad\left(\Re(b)>0 ; \Re(v)>-\frac{1}{2}\right),
$$

and

$$
\begin{aligned}
\int_{0}^{+\infty} K_{2 v}(a \sqrt{x}) K_{v}(b x) d x= & \frac{\pi}{4 b \cos (v \pi)} \times\left(K_{v}\left(\frac{a^{2}}{4 b}\right)+\frac{\pi}{2 \sin (v \pi)}\right. \\
& \left.\times\left[\mathbf{L}_{-v}\left(\frac{a^{2}}{4 b}\right)-L_{v}\left(\frac{a^{2}}{4 b}\right)\right]\right), \quad\left(\Re(b)>0 ;|\Re(v)|<\frac{1}{2}\right) .
\end{aligned}
$$

Finally use the formula

$$
\mathrm{K}_{v}(z)=\frac{\pi}{2} \cdot \frac{\mathrm{I}_{-v}(z)-\mathrm{I}_{v}(z)}{\sin (\pi v)} .
$$

Then the proof is seen to be complete.

Remark 2.4. Other similar formulas as in corollary can be obtained from (2.3).

Further, setting $k=0$ in (2.4) and replacing the Whittaker function $W_{0, \sigma+\frac{1}{2}}$ by Macdonald function $\mathrm{K}_{\sigma+\frac{1}{2}}$, we acquire an interesting integral formula

$$
\int_{0}^{+\infty} \hat{\lambda}^{\sigma+\frac{1}{2}} K_{\sigma+\frac{1}{2}}(\hat{\lambda}) \mathrm{d} \hat{\lambda}=-\frac{2^{\sigma-\frac{1}{2}} \pi^{\frac{3}{2}}}{\sin (\pi \sigma) \Gamma(-\sigma)}
$$

It happens to be seen that, using the following well-known relation (see, e.g., [7, p. 3]):

$$
\Gamma(\sigma) \Gamma(1-\sigma)=\frac{\pi}{\sin (\pi \sigma)}, \quad(\sigma \in \mathbb{C} \backslash \mathbb{Z}),
$$


equation (2.7) is a special case of the following known formula (see, e.g., [1, Entry 6.516.16]):

$$
\int_{0}^{+\infty} x^{\mu} K_{v}(a x) d x=2^{\mu-1} a^{-\mu-1} \Gamma\left(\frac{1+\mu+v}{2}\right) \Gamma\left(\frac{1+\mu-v}{2}\right), \quad(\Re(\mu+1 \pm v) ; \mathfrak{R}(a)>0) .
$$

Yet it is emphasized that the formula (2.7) here is obtained from a group theoretical point of view.

\section{Formulas related to some block-diagonal matrices}

The matrix considered in the previous section can be expressed in terms of $g_{1}$ matrices:

$$
\operatorname{diag}(1,-1,-1)=g_{1}(\pi) .
$$

Here we deal with the matrix

$$
g_{1}\left(\frac{\pi}{2}\right)=\left(\begin{array}{rrr}
1 & 0 & 0 \\
0 & 0 & -1 \\
0 & 1 & 0
\end{array}\right)
$$

Denote the eigenspace of the operator $T^{\bullet}\left(g_{1}\left(\frac{\pi}{2}\right)\right)$ associated with the eigenvalue $\mu$ by $L_{\mu}$. Since $f_{k} \in L_{i^{k}}$, the following equality is valid for the matrix elements of the operator $T^{\bullet}\left(g_{1}\left(\frac{\pi}{2}\right)\right)$ with respect to the basis $\mathrm{B}_{1}$ :

$$
\tilde{\mathrm{t}}_{\mathrm{k} \hat{\mathrm{k}}}\left(g_{1}\left(\frac{\pi}{2}\right)\right)= \begin{cases}\mathbf{i}^{\mathrm{k}}, & \text { if } \mathrm{k}=\hat{\mathrm{k}} \\ 0, & \text { if } \mathrm{k} \neq \hat{\mathrm{k}}\end{cases}
$$

which gives

$$
\mathrm{T}^{\bullet}\left(g_{1}\left(\frac{\pi}{2}\right)\right)\left[\mathrm{f}_{\lambda}^{* *}\right]=\sum_{k \in \mathbb{Z}} \mathbf{i}^{k} c_{\lambda k} f_{k} .
$$

On the other hand, choosing $k=0$ and repeating the reasoning used in the previous section, we have for $\lambda>0$,

$$
\mathrm{T}^{\bullet}\left(\mathrm{g}_{1}\left(\frac{\pi}{2}\right)\right)\left[\mathrm{f}_{\lambda}^{* *}\right]=\sum_{\mathrm{k} \in \mathbb{Z}}\left(\int_{-\infty}^{+\infty} \mathrm{t}_{\lambda \hat{\lambda}}\left(g_{1}\left(\frac{\pi}{2}\right)\right) \mathrm{c}_{\hat{\lambda} k} \mathrm{~d} \hat{\lambda}\right) \mathrm{f}_{\mathrm{k}}
$$

where

$$
\begin{aligned}
t_{\lambda \hat{\lambda}}\left(g_{1}\left(\frac{\pi}{2}\right)\right)= & \frac{\exp (-(\lambda+\hat{\lambda}) \mathbf{i})}{2^{\sigma} \pi} \int_{-\infty}^{+\infty} z^{2 \sigma} \exp \left(\lambda z+\frac{2 \hat{\lambda}}{z}\right) \mathrm{d} z \\
= & \frac{\cos (\pi \sigma)}{\sqrt{2 \pi} \lambda^{\sigma+\frac{1}{2}} \exp (\mathbf{i} \lambda)} \int_{0}^{+\infty} \exp (-\mathbf{i} \hat{\lambda}) \mathrm{K}_{-\sigma-\frac{1}{2}}(\hat{\lambda}) \mathrm{J}_{-2 \sigma-1}(2 \sqrt{2 \lambda \hat{\lambda}}) \mathrm{d} \hat{\lambda} \\
& -\frac{\cos (\pi \sigma)}{\sqrt{2 \pi} \lambda^{\sigma+\frac{1}{2}} \exp (\mathbf{i} \lambda)} \int_{0}^{+\infty} \exp (-\mathbf{i} \hat{\lambda}) \mathrm{K}_{\sigma+\frac{1}{2}}(\hat{\lambda}) \mathrm{J}_{2 \sigma+1}(2 \sqrt{2 \lambda \hat{\lambda}}) \mathrm{d} \hat{\lambda} \\
& +\frac{\sin (\pi \sigma)}{2^{1.5} \pi^{1.5} \lambda^{\sigma+\frac{1}{2}} \exp (\mathbf{i} \lambda)} \int_{0}^{+\infty} \exp (-\mathbf{i} \hat{\lambda}) \mathrm{K}_{\sigma+\frac{1}{2}}(\hat{\lambda}) \mathrm{K}_{2 \sigma+1}(2 \sqrt{2 \lambda \hat{\lambda}}) \mathrm{d} \hat{\lambda} .
\end{aligned}
$$

Expressing the function $K_{\sigma+\frac{1}{2}}(\hat{\lambda}) \equiv K_{-\sigma-\frac{1}{2}}(\hat{\lambda})$ which appears at the integrand of the last formula (2.7) as a series and using the known formulas (see, e.g., [1, Entry 6.643.1 and Entry 6.643.3], respectively): For 
$\mathfrak{R}\left(\mu+v+\frac{1}{2}\right)>0$,

$$
\int_{0}^{+\infty} x^{\mu-\frac{1}{2}} \exp (-\alpha x) J_{2 v}(2 \beta \sqrt{x}) d x=\frac{\Gamma\left(\mu+v+\frac{1}{2}\right)}{\alpha^{\mu} \beta \Gamma(2 v+1)} \exp \left(-\frac{\beta^{2}}{2 \alpha}\right) M_{\mu, v}\left(\frac{\beta^{2}}{\alpha}\right),
$$

and

$$
\int_{0}^{+\infty} x^{\mu-\frac{1}{2}} \exp (-\alpha x) K_{2 v}(2 \beta \sqrt{x}) d x=\frac{\Gamma\left(\mu+v+\frac{1}{2}\right) \Gamma\left(\mu-v+\frac{1}{2}\right)}{2 \alpha^{\mu} \beta} \exp \left(-\frac{\beta^{2}}{2 \alpha}\right) W_{-\mu, v}\left(\frac{\beta^{2}}{\alpha}\right),
$$

we obtain the series $\sum_{n \in \mathbb{Z}}$ containing a linear combination of Whittaker functions

$$
\begin{array}{lll}
M_{2 n-\sigma,-\sigma-\frac{1}{2}}(-2 \mathbf{i} \lambda), & M_{2 n-\sigma, \sigma+\frac{1}{2}}(-2 \mathbf{i} \lambda), & M_{2 n+\sigma+1,-\sigma-\frac{1}{2}}(-2 \mathbf{i} \lambda), \\
M_{2 n+\sigma+1, \sigma+\frac{1}{2}}(-2 \mathbf{i} \lambda), & W_{\sigma-2 n, \sigma+\frac{1}{2}}(-2 \mathbf{i} \lambda), & W_{-2 n-\sigma-1, \sigma+\frac{1}{2}}(-2 \mathbf{i} \lambda),
\end{array}
$$

and converging to the function $K_{\sigma+\frac{1}{2}}(\lambda)$. In the linear combination, we express the Whittaker functions of the first kind in terms of the Whittaker functions of the second kind:

$$
\begin{aligned}
& \frac{\Gamma(2 n+2 \sigma+2)}{\Gamma(2 \sigma+2)} M_{2 n+\sigma+1, \sigma+\frac{1}{2}}(-2 \mathbf{i} \lambda)-\frac{\Gamma(2 n+1)}{\Gamma(-2 \sigma)} M_{2 n+\sigma+1,-\sigma-\frac{1}{2}}(-2 \mathbf{i} \lambda) \\
&=\frac{(2 n) ! \sin (2 \sigma \pi) \Gamma(2 n+2 \sigma+2)}{\pi} \\
& \times\left[\frac{\Gamma(-2 \sigma-1) M_{2 n+\sigma+1, \sigma+\frac{1}{2}}(-2 \mathbf{i} \lambda)}{\Gamma(2 n+1)}+\frac{\Gamma(2 \sigma+1) M_{2 n+\sigma+1,-\sigma-\frac{1}{2}}(-2 \mathbf{i} \lambda)}{\Gamma(2 n+2 \sigma+1)}\right] \\
&= \frac{(2 n) ! \sin (2 \sigma \pi) \Gamma(2 n+2 \sigma+2)}{\pi} W_{2 n+\sigma+1, \sigma+\frac{1}{2}}(-2 \mathbf{i} \lambda),
\end{aligned}
$$

and

$$
\begin{aligned}
& \frac{\Gamma(2 n-2 \sigma)}{\Gamma(-2 \sigma)} M_{2 n-\sigma,-\sigma-\frac{1}{2}}(-2 \mathbf{i} \lambda)-\frac{\Gamma(2 n+1)}{\Gamma(2 \sigma+2)} M_{2 n-\sigma, \sigma+\frac{1}{2}}(-2 \mathbf{i} \lambda) \\
&=-\frac{(2 n) ! \sin (2 \sigma \pi) \Gamma(2 n-2 \sigma)}{\pi} \\
& \times\left[\frac{\Gamma(2 \sigma+1) M_{2 n-\sigma,-\sigma-\frac{1}{2}}(-2 \mathbf{i} \lambda)}{\Gamma(2 n+1)}+\frac{\Gamma(-2 \sigma-1) M_{2 n-\sigma, \sigma+\frac{1}{2}}(-2 \mathbf{i} \lambda)}{\Gamma(2 n-2 \sigma)}\right] \\
&=-\frac{(2 n) ! \sin (2 \sigma \pi) \Gamma(2 n-2 \sigma)}{\pi} W_{2 n-\sigma, \sigma+\frac{1}{2}}(-2 \mathbf{i} \lambda) .
\end{aligned}
$$

Summarizing the argument we have just given, we derive an interesting series representation for $\mathrm{K}_{\sigma+\frac{1}{2}}(\lambda)$ asserted by Theorem 3.1.

Theorem 3.1. For $\lambda>0$,

$$
\begin{aligned}
\mathrm{K}_{\sigma+\frac{1}{2}}(\lambda)= & \frac{\pi^{2}}{2 \Gamma(-\sigma)} \sum_{n=0}^{\infty} \frac{(-1)^{n}(2 n) !}{n !} \\
& \times\left[\frac{2^{-\sigma-2 n-\frac{1}{2}} \mathbf{i}^{-\sigma-1}}{\Gamma\left(\sigma+n+\frac{3}{2}\right)}\left(\frac{\sin (2 \sigma \pi)}{2} W_{2 n+\sigma+1, \sigma+\frac{1}{2}}(-2 \mathbf{i} \lambda)-\frac{\sin (\sigma \pi)}{\pi^{2}} W_{-2 n-\sigma-1, \sigma+\frac{1}{2}}(-2 \mathbf{i} \lambda)\right)\right. \\
& \left.+\frac{2^{\frac{1}{2}+\sigma-2 n} \mathbf{i}^{\sigma}}{\Gamma\left(\frac{1}{2}-\sigma+n\right)}\left(\frac{\sin (\sigma \pi)}{\pi^{2}} W_{\sigma-2 n, \sigma+\frac{1}{2}}(-2 \mathbf{i} \lambda)-\frac{\sin (2 \sigma \pi)}{2} W_{2 n-\sigma, \sigma+\frac{1}{2}}(-2 \mathbf{i} \lambda)\right)\right] .
\end{aligned}
$$


Remark 3.2. Another similar relation as in Theorem 3.1 can be obtained if the matrix $g_{1}\left(\frac{\pi}{2}\right)$ is replaced by the block-diagonal matrix

$$
\mathrm{g}_{3}:=\left(\begin{array}{ccc}
1 & 0 & 0 \\
0 & 0 & 1 \\
0 & 1 & 0
\end{array}\right)
$$

It is clear that, first $g_{3} \in S O(2,1)$ and second for any $k$, the span of the functions $f_{k}$ and $f_{-k}$ is an invariant linear subspace of $\mathfrak{D}^{\bullet}$ with respect to the operator $T\left(g_{3}\right)$ since $T\left(g_{3}\right)^{\bullet}\left[f_{ \pm k}\right]=i^{ \pm k} f_{\mp k}$. By applying the reasoning used here to $T\left(g_{3}\right)^{\bullet}\left[f_{\lambda}^{* *}\right]$, we can obtain a similar result as in Theorem 3.1 whose exact formula and the corresponding details of proof are left to the interested reader.

\section{A series related to the rotation subgroup}

We begin by presenting the matrix elements of the restrictions of the representation to the subgroup of rotations in the plane $x_{2} x_{3}$ asserted by Lemma 4.1. Our result may be a generalization of the cases considered in Sections 2 and 3.

Lemma 4.1. For $\pi<\psi<\frac{3 \pi}{4}$, or $\frac{3 \pi}{2}<\psi<\frac{7 \pi}{4}$,

$$
\begin{aligned}
t_{\lambda \hat{\lambda}}\left(g_{1}(\psi)\right)= & 2^{\sigma+1} \pi^{-\frac{1}{2}} \exp \left(-\mathbf{i} \hat{\lambda} \tan \left[\psi-\frac{\pi}{4}\right]\right) \\
& \times \sum_{n=0}^{\infty} \frac{(-\mathbf{i} \hat{\lambda})^{n}|\lambda|^{n-\sigma-\frac{1}{2}}\left[\tan \left(\psi-\frac{\pi}{4}\right)\right]^{\frac{\sigma-n}{2}+\frac{1}{4}}}{n !\left[\cos \left(\psi-\frac{\pi}{4}\right)\right]^{2 n+1} \Gamma(n-\sigma)} \\
& \left.\times K_{\frac{1}{2}+\sigma-n}\left(\sqrt{\tan \left(\psi-\frac{\pi}{4}\right.}\right)|\lambda|\right) .
\end{aligned}
$$

Proof. We have

$$
\begin{aligned}
t_{\lambda \hat{\lambda}}\left(g_{1}(\psi)\right)= & F_{i}\left(T\left(g_{1}(\psi)\right)\left[f_{\lambda}^{* *}\right], f_{-\hat{\lambda}}^{* *}\right) \\
= & F_{1}\left(f_{\lambda}^{* *}, T^{\bullet}\left(g_{1}(-\psi)\right)\left[f_{-\hat{\lambda}}^{* *}\right]\right) \\
= & \frac{\exp \left(\mathbf{i} \hat{\lambda} \tan \left[\frac{\pi}{4}-\psi\right]\right)}{2^{2.5} \pi} \\
& \times \int_{-\infty}^{+\infty} \exp (\mathbf{i} \lambda y)\left(y^{2}+\tan \left[\frac{\pi}{4}-\psi\right]\right)^{\sigma} \exp \frac{-\mathbf{i} \hat{\lambda}\left[\sec \left(\psi-\frac{\pi}{4}\right)\right]^{2}}{y^{2}+\tan \left[\frac{\pi}{4}-\psi\right]} d y
\end{aligned}
$$

Then expand the function

$$
\exp \frac{-\mathbf{i} \hat{\lambda}\left[\sec \left(\psi-\frac{\pi}{4}\right)\right]^{2}}{y^{2}+\tan \left[\frac{\pi}{4}-\psi\right]}
$$

as the Taylor series and use the following known formula (see, e.g., [2, Entry 2.5.6.4]):

$$
\int_{0}^{+\infty} \frac{\cos (b x) d x}{\left(x^{2}+z^{2}\right)^{\rho}}=\left(\frac{2 z}{b}\right)^{\frac{1}{2}-\rho} \frac{\sqrt{\pi}}{\Gamma(\rho)} K_{\frac{1}{2}-\rho}(b z), \quad(\min \{b, \mathfrak{R}(\rho), \mathfrak{R}(z)>0\}>0) .
$$

Lemma 4.2. For $\pi<\psi<\frac{3 \pi}{4}$, or $\frac{3 \pi}{2}<\psi<\frac{7 \pi}{4}$,

$$
\frac{2^{\sigma+\frac{1}{2}}}{\sqrt{\pi}} \sum_{n=0}^{\infty} \frac{(-\mathbf{i})^{n}|\lambda|^{n+\frac{1}{2}}\left[\tan \left(\psi-\frac{\pi}{4}\right)\right]^{\frac{\sigma-n}{2}+\frac{1}{4}}}{n !\left[\cos \left(\psi-\frac{\pi}{4}\right)\right]^{2 n+1} \Gamma(n-\sigma)} K_{\frac{1}{2}+\sigma-n}\left(\sqrt{\tan \left(\psi-\frac{\pi}{4}\right)}|\lambda|\right)
$$




$$
\begin{aligned}
& \times\left[\int_{0}^{+\infty} \hat{\lambda}^{n-\sigma-1} \exp \left(-\mathbf{i} \hat{\lambda} \tan \left[\psi-\frac{\pi}{4}\right]\right) W_{-k, \sigma+\frac{1}{2}}(2 \hat{\lambda}) \mathrm{d} \hat{\lambda}\right. \\
& \left.+(-1)^{\mathrm{n}} \int_{0}^{+\infty} \hat{\lambda}^{\mathrm{n}-\sigma-1} \exp \left(\mathbf{i} \hat{\lambda} \tan \left[\psi-\frac{\pi}{4}\right]\right) W_{-k, \sigma+\frac{1}{2}}(2 \hat{\lambda}) \mathrm{d} \hat{\lambda}\right] \\
& =\exp (-\mathbf{i} \psi k)[\Gamma(-\mathrm{k} \operatorname{sign} \lambda-\sigma)]^{-1} W_{-k \operatorname{sign} \lambda, \sigma+\frac{1}{2}}(2|\lambda|) .
\end{aligned}
$$

Proof. Let $A(k)$ denote the coefficient of the distribution of the function $T^{\bullet}\left(g_{1}(\psi)\right)\left[f_{\lambda}^{* *}(x)\right]$ with respect to the basis $B_{1}$ in the equality

$$
\begin{aligned}
T^{\bullet}\left(g_{1}(\psi)\right)\left[f_{\lambda}^{* *}(x)\right] & =\int_{-\infty}^{+\infty} t_{\lambda \hat{\lambda}}\left(g_{1}(\psi)\right) f_{\hat{\lambda}}^{* *}(x) d \hat{\lambda} \\
& =\sum_{k \in \mathbb{Z}}\left(\int_{-\infty}^{+\infty} t_{\lambda \hat{\lambda}}\left(g_{1}(\psi)\right) c_{\hat{\lambda} k} d \hat{\lambda}\right) f_{k}(x)
\end{aligned}
$$

Let $U_{\exp (-i \psi k)}$ be the eigenspace of $T^{\bullet}\left(g_{3}\right)$ associated with the eigenvalue $\exp (-\mathbf{i} \psi k)$. It is easy to see that $f_{k} \in U_{\exp (-i \psi k)}$ and therefore, the 'matrix' of $T^{\bullet}\left(g_{3}\right)$ with respect to the basis $B_{1}$ is diagonal. From the equality

$$
\begin{aligned}
T^{\bullet}\left(g_{1}(\psi)\right)\left[f_{\lambda}^{* *}\right] & =T^{\bullet}\left(g_{1}(\psi)\right)\left[\sum_{k \in \mathbb{Z}} c_{\lambda k} f_{k}\right] \\
& =\sum_{k \in \mathbb{Z}} c_{\lambda k} T^{\bullet}\left(g_{1}(\psi)\right)\left[f_{k}\right]=\sum_{k \in \mathbb{Z}} \exp (-\mathbf{i} \psi k) c_{\lambda k} f_{k},
\end{aligned}
$$

we have $A(k)=\exp (-\mathbf{i} \psi k) c_{\lambda k}$ and use the result in Lemma 2.1.

Putting $k=0$ in Lemma 4.1 and expressing the Whittaker function in terms of Macdonald function and using the known formula (see, e.g., [1, Entry 6.621.3]):

$$
\begin{aligned}
\int_{0}^{+\infty} x^{\mu-1} \exp (-\alpha x) K_{v}(\beta x) d x= & \frac{\sqrt{\pi}(2 \beta)^{v}}{(\alpha+\beta)^{v+\mu}} \frac{\Gamma(\mu+v) \Gamma(v-\mu)}{\Gamma\left(\mu+\frac{1}{2}\right)} \\
& \times{ }_{2} F_{1}\left(\mu+v, v+\frac{1}{2} ; \mu+\frac{1}{2} ; \frac{\alpha-\beta}{\alpha+\beta}\right), \quad(\mathfrak{R}(\mu)>|\mathfrak{R}(\nu)| ; \Re(\alpha+\beta)>0),
\end{aligned}
$$

we obtain the following interesting representation for $\mathrm{K}_{\sigma+\frac{1}{2}}(2 \lambda)$ given in Theorem 4.3.

Theorem 4.3. For $\lambda>0$ and $\frac{\pi}{4}<\psi<\pi$, or $\frac{5 \pi}{4}<\psi<\frac{3 \pi}{2}$,

$$
\begin{aligned}
2^{\sigma+\frac{1}{2}} \sum_{n=0}^{\infty} & \frac{(-\mathbf{i})^{n} \lambda^{n}[\tan \psi]^{\frac{\sigma-n}{2}+\frac{1}{4}} \Gamma(n+1) \Gamma(n-2 \sigma)}{n ![\cos \psi]^{2 n+1}(-\sigma)_{n}(-\sigma)_{n+1}} \\
& \times K_{\frac{1}{2}+\sigma-n}(\sqrt{\tan \psi} \lambda)\left[\frac{{ }_{2} F_{1}\left(n+1, \sigma+1 ; 1+n-\sigma ; \frac{i \tan \psi-1}{i \tan \psi+1}\right)}{(1+i \tan \psi)^{n+1}}\right. \\
& \left.+\frac{{ }_{2} F_{1}\left(n+1, \sigma+1 ; 1+n-\sigma ; \frac{i \tan \psi+1}{i \tan \psi-1}\right)}{(1-i \tan \psi)^{n+1}}\right]=K_{\sigma+\frac{1}{2}}(2 \lambda) .
\end{aligned}
$$




\section{Some relations between Bessel and Struve functions related to the matrix $\operatorname{diag}(1,-1,1)$}

Let us express the function $f_{\lambda}^{* *} \in \mathfrak{D}^{\bullet}$ as an 'infinite continuous linear combination' of the functions belonging to the basis $B_{2}$ in $\mathfrak{D}^{\bullet}$ :

$$
f_{\lambda}^{* *}(x)=\int_{-\infty}^{+\infty} d_{\lambda, \rho,+} f_{\rho,+}^{*}(x) d \rho+\int_{-\infty}^{+\infty} d_{\lambda, \rho,-} f_{\rho,-}^{*}(x) d \rho .
$$

Then we find

$$
\begin{aligned}
\frac{1}{2 \pi} F_{i}\left(f_{\lambda}^{* *}, f_{\tau, \pm}^{*}\right) & =\frac{1}{2 \pi} \int_{-\infty}^{+\infty} d_{\lambda, \rho, \pm} F_{2}\left(f_{\rho, \pm}^{*} f_{\tau, \pm}^{*}\right) d \rho \\
& =\frac{1}{2 \pi} \int_{-\infty}^{+\infty} d_{\lambda, \rho, \pm} \int_{-\infty}^{+\infty} \exp (\mathbf{i}[\rho+\tau] s) d s d \rho=d_{\lambda,-\tau, \pm}
\end{aligned}
$$

We therefore get $d_{\lambda, \rho, \pm}=\frac{1}{2 \pi} F_{i}\left(f_{\lambda}^{* *}, f_{-\rho, \pm}^{*}\right)$.

\section{Lemma 5.1.}

$$
\mathrm{d}_{\lambda, 0,+}=\frac{2^{2 \sigma+\frac{1}{2}} \Gamma\left(\sigma+\frac{3}{2}\right) \mathrm{B}(\sigma+1, \sigma+1)}{\pi \lambda^{\sigma+\frac{1}{2}}} \mathrm{~J}_{\sigma+\frac{1}{2}}(\lambda),
$$

and

$$
\mathrm{d}_{\lambda, 0,-}=-\frac{\Gamma(\sigma+1)}{\sqrt{2 \pi} \lambda^{\sigma+\frac{1}{2}}} Y_{-\sigma-\frac{1}{2}}(\lambda)
$$

Proof. We have

$$
\begin{aligned}
d_{\lambda, \rho,+}=\frac{1}{2 \pi} F_{2}\left(f_{\lambda}^{* *}, f_{-\rho,+}\right) & =\frac{2^{-\sigma-1}}{\pi} \int_{-1}^{1}(1-y)^{\sigma+\mathbf{i} \rho}(1+y)^{\sigma-\mathbf{i} \rho} \exp (\mathbf{i} \lambda y) d y \\
& =\frac{\exp (\mathbf{i} \lambda)}{2^{\sigma+1} \pi} \int_{0}^{2} t^{\sigma+\mathbf{i} \rho}(2-t)^{\sigma-\mathbf{i} \rho} \exp (-\mathbf{i} \lambda t) d t
\end{aligned}
$$

which can be computed by recalling the following known formula (see, e.g., [2, Entry 2.3.6.1]):

$$
\int_{0}^{a} x^{\alpha-1}(a-x)^{\beta-1} \exp (-p x) d x=a^{\alpha+\beta-1} B(\alpha, \beta){ }_{1} F_{1}(\alpha ; \alpha+\beta ;-a p), \quad(\mathfrak{R}(\alpha)>0 ; \mathfrak{R}(\beta)>0) .
$$

Applying the following relations:

$$
\begin{gathered}
{ }_{1} \mathrm{~F}_{1}\left(\alpha-\beta+\frac{1}{2} ; 2 \alpha+1 ; x\right)=x^{-\alpha-\frac{1}{2}} \exp \left(\frac{x}{2}\right) M_{\beta, \alpha}(x), \\
M_{0, v}(x)=2^{2 v} \Gamma(v+1) \sqrt{x} I_{v}\left(\frac{x}{2}\right),
\end{gathered}
$$

and

$$
\mathrm{J}_{v}(\mathrm{x})=\exp \left(\frac{\mathbf{i} \pi v}{2}\right) \mathrm{I}_{v}(-\mathbf{i} x),
$$

to the resulting identity, we are led to the first one of the two formulas in this lemma. 
On the other hand, starting with the following expression:

$$
\begin{aligned}
d_{\lambda, 0,-} & =\frac{2^{-\sigma-1}}{\pi}\left[\int_{1}^{+\infty}\left(y^{2}-1\right)^{\sigma} \exp (\mathbf{i} \lambda y) d y+\int_{-\infty}^{-1}\left(y^{2}-1\right)^{\sigma} \exp (\mathbf{i} \lambda y) d y\right] \\
& =\frac{2^{-\sigma}}{\pi} \int_{1}^{+\infty}\left(y^{2}-1\right)^{\sigma} \cos (\lambda y) d y,
\end{aligned}
$$

and using a known formula (see, e.g., [2, Entry 2.5.6.2]):

$$
\int_{a}^{+\infty}\left(x^{2}-a^{2}\right)^{\beta-1} \cos (b x) d x=-\frac{\sqrt{\pi}}{2}\left(\frac{2 a}{b}\right)^{\beta-\frac{1}{2}} \Gamma(\beta) Y_{\frac{1}{2}-\beta}(a b), \quad(\min \{a, b\}>0 ; 0<\mathfrak{R}(\beta)<1),
$$

similarly as above, we prove the second formula.

Remark 5.2. The span of the functions $f_{\rho,+}^{*}$ and $f_{\rho,-}^{*}$ in the linear space $\mathfrak{D}^{\bullet}$ is invariant with respect to the linear operator $T^{\bullet}(\operatorname{diag}(1,-1,1))$, because its image of any linear combination $\alpha f_{\rho,+}^{*}+\beta f_{\rho,-}^{*}$ is the linear combination $\beta f_{\rho,+}^{*}+\alpha f_{\rho,-}^{*}$. It implies that

$$
\begin{aligned}
\mathrm{T}^{\bullet}(\operatorname{diag}(1,-1,1))\left[\mathrm{f}_{\lambda}^{* *}\right] & =\mathrm{T} \bullet(\operatorname{diag}(1,-1,1))\left[\int_{-\infty}^{+\infty} \mathrm{d}_{\lambda, \rho,+} \mathrm{f}_{\rho,+}^{*} \mathrm{~d} \rho+\int_{-\infty}^{+\infty} \mathrm{d}_{\lambda, \rho,-} f_{\rho,-}^{*} \mathrm{~d} \rho\right] \\
& =\int_{-\infty}^{+\infty} \mathrm{d}_{\lambda, \rho,+} \mathrm{f}_{\rho,-}^{*} \mathrm{~d} \rho+\int_{-\infty}^{+\infty} \mathrm{d}_{\lambda, \rho,-} \mathrm{f}_{\rho,+}^{*} \mathrm{~d} \rho .
\end{aligned}
$$

On the other hand, we have

$$
\begin{aligned}
T^{\bullet}(\operatorname{diag}(1,-1,1))\left[f_{\lambda}^{* *}\right]= & \int_{-\infty}^{+\infty} t_{\lambda \hat{\lambda}}(\operatorname{diag}(1,-1,1)) f_{\hat{\lambda}}^{* *} d \hat{\lambda} \\
= & \int_{-\infty}^{+\infty}\left(\int_{-\infty}^{+\infty} t_{\lambda \hat{\lambda}}(\operatorname{diag}(1,-1,1)) d_{\hat{\lambda}, \rho,+} d \hat{\lambda}\right) f_{\rho,+}^{*} d \rho \\
& +\int_{-\infty}^{+\infty}\left(\int_{-\infty}^{+\infty} t_{\lambda \hat{\lambda}}(\operatorname{diag}(1,-1,1)) d_{\hat{\lambda}, \rho,-} d \hat{\lambda}\right) f_{\rho,-}^{*} d \rho
\end{aligned}
$$

It implies that, in particular,

$$
d_{\lambda, 0, \pm}=\int_{-\infty}^{+\infty} t_{\lambda \hat{\lambda}}(\operatorname{diag}(1,-1,1)) d_{\hat{\lambda}, \rho, \mp} d \hat{\lambda}
$$

Lemma 5.3. The following relations hold true:

$\lambda \hat{\lambda}>0$.

$$
\mathrm{t}_{\lambda \hat{\lambda}}(\operatorname{diag}(1,-1,1))=-\frac{2 \sin (\pi \sigma)}{\pi}\left(\frac{\hat{\lambda}}{\lambda}\right)^{\sigma+\frac{1}{2}} \mathrm{~K}_{2 \sigma+1}(2 \sqrt{\lambda \hat{\lambda}})
$$

- $\lambda \hat{\lambda}<0$.

$$
t_{\lambda \hat{\lambda}}(\operatorname{diag}(1,-1,1))=\frac{\cos (\pi \sigma)}{2}\left(\frac{-\hat{\lambda}}{\lambda}\right)^{\sigma+\frac{1}{2}}\left[J_{-2 \sigma-1}(2 \sqrt{-\lambda \hat{\lambda}})-J_{2 \sigma+1}(2 \sqrt{-\lambda \hat{\lambda}})\right]
$$


From a group theoretical point of view, the upper part of the equality (5.1) allows to write an interesting relation asserted by the following theorem.

Theorem 5.4. The following relation holds true:

$$
\begin{aligned}
J_{\sigma+\frac{1}{2}}(\lambda)= & \frac{1}{1-\sin (\pi \sigma)}\left[\left(\frac{2^{-2 \sigma} \sqrt{\pi} \Gamma(2 \sigma+2)}{\Gamma(\sigma+1) \Gamma\left(\sigma+\frac{3}{2}\right)}+2 \cot (\pi \sigma)+(-1)^{\sigma+\frac{1}{2}}\right) Y_{-\sigma-\frac{1}{2}}(\lambda)\right. \\
& \left.-\cos (\pi \sigma) Y_{-\sigma+\frac{1}{2}}(\lambda)+\left((-1)^{\sigma+\frac{1}{2}}-2 \tan (\pi \sigma)\right) \mathbf{H}_{-\sigma-\frac{1}{2}}(\lambda)\right], \quad(\lambda>0) .
\end{aligned}
$$

Proof. For the integral $\int_{0}^{+\infty} t_{\lambda \hat{\lambda}}(\operatorname{diag}(1,-1,1)) d_{\hat{\lambda}, \rho,+} d \hat{\lambda}$, we use the following formula (see, e.g., [1, Entry 6.516.7]):

$$
\begin{gathered}
\int_{0}^{+\infty} \mathrm{K}_{2 v}(\mathrm{a} \sqrt{x}) J_{v}(b x) d x=\frac{\pi \sec (v \pi)}{4 b} \times\left[\mathbf{H}_{-v}\left(\frac{a^{2}}{4 b}\right)-Y_{-v}\left(\frac{a^{2}}{4 b}\right)\right], \\
(\min \{\mathfrak{R}(a), \mathfrak{R}(b)\}>0 ; \mathfrak{R}(v)>-1 / 2) .
\end{gathered}
$$

For the integral $\int_{-\infty}^{0} t_{\lambda \hat{\lambda}}(\operatorname{diag}(1,-1,1)) d_{\hat{\lambda}, \rho,+} d \hat{\lambda}$, we use the following formulas (see, e.g., [1, Entry 6.516.1] and [1, 6.516.4], respectively):

$$
\int_{0}^{+\infty} J_{2 v}(a \sqrt{x}) J_{v}(b x) d x=b_{-1} J_{v}\left(\frac{a^{2}}{4 b}\right), \quad(\min \{\mathfrak{R}(a), \mathfrak{R}(b)\}>0 ; \mathfrak{R}(v)>-1 / 2),
$$

and

$$
\begin{gathered}
\int_{0}^{+\infty} \mathrm{Y}_{2 v}(\mathrm{a} \sqrt{x}) J_{v}(b x) d x=\frac{2 \sec (v \pi)}{b}\left[\frac{\cos (v \pi)}{2} Y_{v}\left(\frac{a^{2}}{4 b}\right)-Y_{-v}\left(\frac{a^{2}}{4 b}\right)+H_{-v}\left(\frac{a^{2}}{4 b}\right)\right], \\
(\min \{\mathfrak{R}(a), \mathfrak{R}(b)\}>0 ; \mathfrak{R}(v)>-1 / 2) .
\end{gathered}
$$

Finally using

$$
J_{-v}(x)=\cos (\pi v) J_{v}(x)-\sin (\pi v) Y_{v}(x),
$$

we are led to the desired result.

It is also remarked that a similar argument applied to the lower part of (5.1) can establish another relation as in Theorem 5.4.

\section{Concluding Remarks}

It seems to be obvious that some other interesting formulas can be obtained by considering the similar construction for special orthogonal groups of higher dimension. For instance, very recently we have expressed some matrix elements of the representation operators for $\mathrm{SO}(3,1)$ in terms of the modified hyper Bessel functions of the first kind, which is a multi-index generalization of classical eponymous functions. In particular, it yielded a formula for integral of the product an ordinary Macdonald function and its multi-index analogue. The real presentation of further related results may be left to the interested researcher. It is emphasized again that we have shown how certain interesting formulas for some classical and useful special functions can be nicely obtained from a group theoretical point of view. 


\section{References}

[1] I. S. Gradshteyn, I. M. Ryzhik, Table of Integrals, Series, and Products, Translated from the Russian. Translation edited and with a preface by Alan Jeffrey and Daniel Zwillinger., Academic Press, Amsterdam, (2007). 2, 2, 3, 4, 5

[2] A. P. Prudnikov, Yu. A. Brychkov, O. I. Marichev, Integrals and Series, Vol. 1: Elementary Functions, OPA (Overseas Publishers Association), Amsterdam B. V. Published under the license of Gordon and Breach Science Publishers, New York, (1986). 2, 2, 4, 5

[3] I. A. Shilin, Double SO(2,1)-integrals and formulas for Whittaker functions, Russian Math., 56 (2012), 47-56. 1, 2

[4] I. A. Shilin, J. S. Choi, Certain connections between the spherical and hyperbolic bases on the cone and formulas for related special functions, Integral Transforms Spec. Funct., 25 (2014), 374-383.1

[5] I. A. Shilin, J. Choi, Some connections between the spherical and parabolic bases on the cone expressed in terms of the Macdonald function, Abstr. Appl. Anal., 2014 (2014), 8 page.

[6] I. A. Shilin, J. Choi, Transformations of bases related to the six-dimensional split orthogonal group and special functions, (submitted). 1

[7] H. M. Srivastava, J. Choi, Zeta and q-Zeta Functions and Associated Series and Integrals, Elsevier Science Publishers, Amsterdam, (2012). 2

[8] N. J. Vilenkin, M. A. Sleinikova, Integral relations for the Whittakers functions and the representations of the threedimensional Lorentz group, Math. USSR Sb., 10 (1970), 173-180. 1, 2, 2 